\title{
Ezetimibe for the treatment of primary hypercholesterolaemia
}

\author{
Katherine Croom \\ CSF Medical Communications, Cheltenham, UK
}

Drugs in Context

DOI: http://dx.doi.org/10.7573/dic.212223

Citation: Croom, K. Ezetimibe for the treatment of primary hypercholesterolaemia. Drugs in Context: e212223. doi:10.7573/dic.212223

Copyright: this is an open access article published under the terms of the Creative Commons License Deed (CG BY-NC-ND 3.0) which allows you to share, copy, distribute and transmit the work provided it is properly attributed. You may not use this work for commercial purposes. For further information on commercial use, contact publisher@justmedicalmedia.com or go to wrwre.drugsincontext.com/copyright.

Search criteria: English language articles were identified by searching the PubMed database using the search term 'ezetimibe'. Abstracts were evaluated and selected for further review according to our standard protocols. Bibliographies of individual articles were also assessed for additional articles of interest and the manufacturer of ezetimibe was invited to supply any additional data to that identified via the PubMed database.

Date of last literature search: 1 July 2008.

Conclusion: Ezetimibe treatment allows more patients to reach their target LDL-C level, whether given in combination with other lipid modifying therapies or as monotherapy for patients who cannot tolerate statins. The National Institute for Health and Clinical Excellence for England and Wales (NICE) recommends coadministering ezetimibe instead of switching from initial statin therapy. The NICE Committee agrees that this treatment option is a cost-effective use of NHS resources when compared with switching to an alternative statin.

NB: This article was originally published by CSF Medical Communications Ltd (CSF) in Drugs in Context 2008;4(2):153-72. Drugs in Context and all CSF copyrights were acquired by Just Medical Media Ltd in 2009. 


\section{SUMMARY}

Following a decision to commence drug treatment for the primary or secondary prevention of cardiovascular disease (GVD), simvastatin, $40 \mathrm{mg}$, is recommended first for most patients. However, some people may require additional or alternative treatment in order to modify their lipid profile and reach recommended target levels. Many patients treated with statins still fail to achieve increasingly stringent LDL-C goals in clinical practice, and can benefit from add-on ezetimibe therapy, which has a complementary mechanism of action to other drugs in current use. Many clinical studies have demonstrated that combinations of ezetimibe with statins, fibrates, and other therapies, provide better modification of lipid profiles than these drugs given as monotherapy. Moreover, ezetimibe is also effective as a monotherapy. The safety and tolerability profile of ezetimibe as monotherapy is similar to placebo, and is also excellent when given in combination with other lipid-modifying agents. Ezetimibe may also provide a cost-effective alternative to statin titration. These findings suggest that ezetimibe represents a useful alternative and adjunct to other lipid management strategies.

Key words: ezetimibe; Ezetrol; hypercholesterolaemia; hyperlipidaemia.

\section{HYPERCHOLESTEROLAEMIA: A PERSPECTIVE}

High low-density lipoprotein (LDL-C) is a wellestablished risk factor for the development of cardiovascular disease, and is the main target for cholesterol- and lipid-lowering therapy. The risk of coronary heart disease (CHD) - one of the main components of cardiovascular diseaseincreases with increasing plasma cholesterol levels, with a $\log$-linear relationship apparent between plasma levels of LDL-C and the relative risk for CHD. ${ }^{1}$ Conversely, cardiovascular risk is inversely related to levels of high density lipoprotein cholesterol (HDL-C). Cardiovascular disease continues to be the major cause of mortality, and is responsible for about $36 \%$ of all deaths in the UK alone. ${ }^{2}$

Statins - which work primarily by decreasing cholesterol synthesis - are the most commonly used therapies for patients with hypercholesterolaemia. A number of landmark studies have elegantly demonstrated that changes to the lipid profile elicited by long-term statin treatment translate into real benefits in terms of cardiovascular outcomes. ${ }^{3,4}$ Despite the established efficacy of statins for improving lipid profiles and reducing cardiovascular risk, a substantial proportion of patients fail to meet LDL-C goals set out in various national and international cholesterol management guidelines. ${ }^{5,6}$ This is primarily because of an inadequate response to therapy, poor compliance or concerns about the increased potential for side effects at higher doses. ${ }^{5,7,8}$ Moreover, the most recent guidelines from the National Cholesterol Education Program (NCEP) in the USA have established even more stringent LDL-G goals, particularly for patients with established CHD or in those with CHD risk equivalents, such as those with type 2 diabetes. ${ }^{6}$ As the evidence base continues to support increasingly aggressive lipid lowering, particularly in very high-risk patients (i.e. those suffering acute cardiovascular events or with existing cardiovascular disease plus other risk factors), the NCEP guidelines have been modified to include a new optional lower target for such patients of under $70 \mathrm{mg} / \mathrm{dL}$ (equivalent to $\left.1.8 \mathrm{mmol} / \mathrm{L}^{\mathrm{a}}\right){ }^{1}$

Alternative lipid-modifying agents are available which have distinct and complementary mechanisms of action to

aThroughout this review we have reported cholesterol concentrations (including US guideline targets) in SI units. 
the statins. Ezetimibe is one such treatment, acting to selectively inhibit dietary and biliary cholesterol absorption across the wall of the small intestine, thereby reducing delivery of intestinal cholesterol to the liver. Thus, ezetimibe can be used instead of statins for patients who are intolerant to statins or where uptitration fails to meet treatment targets. Moreover, ezetimibe can be given as addon therapy in patients already receiving a statin, thus inhibiting both the exogenous and endogenous pathways of cholesterol generation, resulting in greater reductions in cholesterol levels compared with statin or ezetimibe monotherapy. ${ }^{5,9}$ By combining ezetimibe and a statin, the requirement for statin dose titration can be reduced, thereby minimising the likelihood of dose-related adverse events.

Ezetimibe is also available as a fixed-dose combination (FDC) product that combines ezetimibe $(10 \mathrm{mg})$ with varying doses of simvastatin (20, 40 and $80 \mathrm{mg}$ ) in a single tablet. This FDC is the subject of a separate Drugs in Context - Drug Profile. ${ }^{10}$ This review of ezetimibe examines the clinical and pharmacological evidence that has accumulated for ezetimibe monotherapy and as add-on or combination therapy with other lipid-modifying therapies including statins other than simvastatin (which is covered in the aforementioned FDC article).

\section{PHARMACOLOGY}

\section{Mechanism of action}

Ezetimibe is the first in a novel class of cholesterol absorption inhibitors, the 2-azetidinones, and was synthetically developed from a parent compound on the basis of structureactivity relationships. ${ }^{11,12}$ Ezetimibe and its active glucuronidated metabolite localise to the brush border of the small intestine and are thought to block the actions of a cholesterol transport protein, which until recently was believed to be the Niemann-Pick G1 Like 1 protein [NPG1L1]). ${ }^{13}$
However, recent knock-out experiments in mice have challenged the prevailing belief that NPG1L1 facilitates intestinal cholesterol uptake into the brush border membrane and that the inhibition of NPC1L1 by ezetimibe is responsible for the inhibition of this process. ${ }^{14}$ Although the mechanism of action remains to be fully elucidated, the net effect of ezetimibe therapy is to inhibit the absorption of dietary and biliary cholesterol across the intestinal wall (by about $50 \%$ ) without affecting the absorption of triglycerides, fatty acids, bile acids, progesterone and ethinyl estradiol, or fat-soluble vitamins. ${ }^{15}$ Consequently, delivery of cholesterol to the liver is reduced, resulting in a reduction in hepatic cholesterol stores and an increase in cholesterol clearance from the circulation.

\section{Pharmacokinetics}

Ezetimibe does not have significant effects on plasma levels of any other statins (atorvastatin, fluvastatin, lovastatin, pitavastatin, pravastatin, rosuvastatin or simvastatin), fibric acidderivatives (gemfibrozil or fenofibrate), digoxin, glipizide, warfarin or triphasic oral contraceptives (ethinyl estradiol and levonorgestrel). ${ }^{16}$ Concomitant administration of food, antacids, cimetidine or statins has no significant effect on ezetimibe bioavailability, but coadministration with gemfibrozil and fenofibrate increases the bioavailability of ezetimibe, though this was not considered to be clinically significant. ${ }^{16,17}$ In contrast, coadministration of ezetimibe with colestyramine significantly decreased ezetimibe oral bioavailability, so ezetimibe and colestyramine should be taken several hours apart to avoid attenuating the efficacy of ezetimibe. ${ }^{16}$ Higher ezetimibe exposures have been observed in patients receiving concomitant ciclosporin, and ezetimibe caused a small but statistically significant effect on plasma levels of ciclosporin, and because treatment experience in this group is limited, physicians should be 
cautious when starting ezetimibe therapy in the setting of ciclosporin coadministration, and careful monitoring of ciclosporin levels should be undertaken. ${ }^{16}$

The principal pharmacokinetic parameters of ezetimibe and its glucuronidated metabolite are summarised in Table 1.,18 Ezetimibe is rapidly absorbed and undergoes rapid and extensive glucuronidation in the intestine to form the active metabolite. ${ }^{9,18}$ Peak plasma concentrations are reached 1-2 hours after dosing for the active glucuronidated metabolite and after 4-12 hours for the parent compound, and it has a long terminal half-life which allows once-daily dosing. ${ }^{16,18}$ Ezetimibe glucuronide undergoes repeated enterohepatic recirculation, delivering drug back to the intestinal lumen via bile, thereby prolonging the effect of the original dose and potentiating ezetimibe's lipidmodifying effects. ${ }^{19}$ Ezetimibe is not metabolised by the cytochrome P450 (CYP) system in the liver, and therefore has a low propensity to interact with other drugs. ${ }^{20}$

\section{Dosing}

Ezetimibe is given once daily as a $10 \mathrm{mg}$ tablet. The pharmacokinetic characteristics of ezetimibe are similar for children/adolescents (10-18 years)

Table 1. The pharmacokinetic characteristics of ezetimibe and its glucuronidated metabolite. ${ }^{9,18}$

\begin{tabular}{|c|c|c|}
\hline $\begin{array}{l}\text { Pharmacokinetic } \\
\text { parameter }\end{array}$ & Ezetimibe & $\begin{array}{l}\text { Ezetimibe- } \\
\text { glucuronide }\end{array}$ \\
\hline Volume of distribution (L) & $105-107.5$ & - \\
\hline$t_{\max }$ (hours) & $4-12$ & $1-2$ \\
\hline $\mathrm{t}_{1 / 2}$ (hours) & $\sim 22$ & $\sim 22$ \\
\hline AUC (mg/L/hour) & - & 10 \\
\hline $\mathrm{C}_{\max }(\mu \mathrm{g} / \mathrm{L})$ & $70.6-79.3$ & 2.6 \\
\hline Protein binding (\%) & 99.7 & 88-92 \\
\hline Metabolism & Glucuronidation & - \\
\hline Elimination in urine/faeces (\%) & $11 / 78$ & - \\
\hline
\end{tabular}

and adults, so no dosage adjustments are required in this age group. ${ }^{18}$ Although plasma concentrations are about two-fold higher in elderly patients (at least 65 years) than younger patients, safety profiles are similar for these groups, so no dosage adjustments are needed in the elderly. ${ }^{18}$ Moreover, no dose adjustments are required in patients with mild hepatic impairment (ChildPugh score 5 or 6 ) or those with renal impairment, but exposure to ezetimibe is increased four-fold in patients with moderate hepatic insufficiency (Child-Pugh score 7-9) and so ezetimibe is not recommended for patients with moderate or severe hepatic insufficiency. ${ }^{18}$

\section{CLINICAL EFFICACY}

A number of studies have evaluated the lipid-modifying effects of ezetimibe, both as monotherapy and in addition to other treatments. Here, we will discuss studies that evaluated ezetimibe monotherapy, and trials investigating the combination of ezetimibe plus other lipidmodifying treatments (with the exception of simvastatin/ezetimibe combinations which have been covered in a separate Drugs in Context - Drug Profile $\left.{ }^{10}\right)$.

\section{Monotherapy: placebo-controlled studies}

Two companion, multicentre, randomised, double-blind studies have investigated the efficacy and safety of ezetimibe, $10 \mathrm{mg} /$ day, compared with placebo in large populations of patients $(n=1719)$ with primary hypercholesterolaemia. ${ }^{21,22}$ Both studies were of 12 weeks' duration, and had identical primary endpoints (percentage reduction in LDL-C from baseline to study end). Patients were also required to have similar baseline LDL-C and triglyceride levels $(3.4-6.5 \mathrm{mmol} / \mathrm{L}$ and $<4.0 \mathrm{mmol} / \mathrm{L}$, respectively). In the first study, 892 patients were given ezetimibe or placebo, and LDL-C levels were reduced by $16.9 \%$ from baseline compared with an increase of 
Figure 1. Onset of low-density lipoprotein cholesterol (LDL-C) reductions in patients given ezetimibe, $10 \mathrm{mg}$ once daily, or placebo, during a 12-week study. ${ }^{21}$

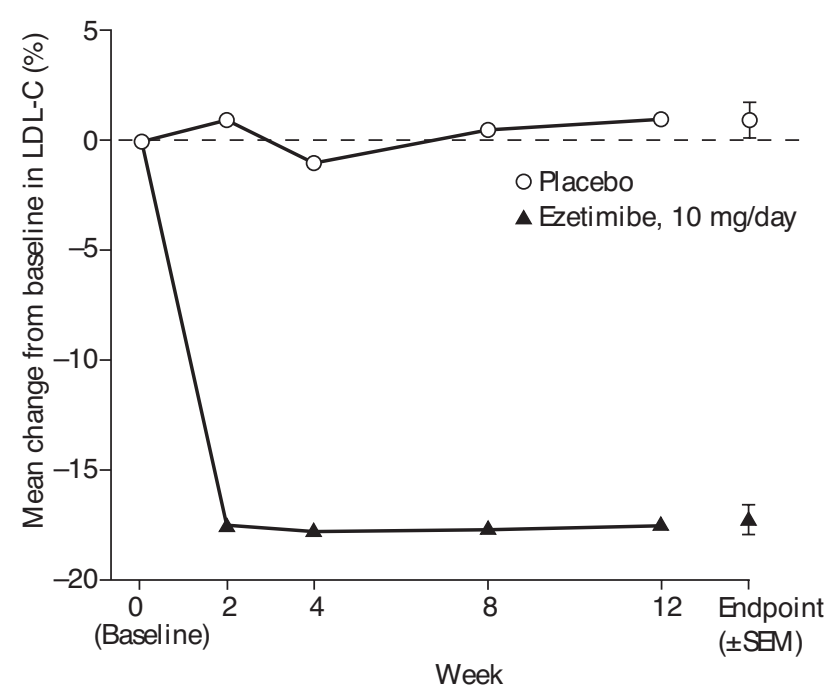

$0.4 \%$ in the placebo group $(p<0.01){ }^{21}$ The second study showed LDL-G reductions of $17.7 \%$ for the ezetimibe group compared with an increase of $0.8 \%$ for the placebo group $(p<0.01)$, with LDL-G reductions occurring within 2 weeks of starting therapy and being maintained until the end of the trial (Figure
1). ${ }^{21}$ In addition, ezetimibe favourably modified other lipid profile components, increasing HDL-C, whilst reducing apolipoprotein B (Apo B; the principle protein component of LDL-C) and reducing total cholesterol levels (Table 2). ${ }^{21,22}$ Triglyceride levels were reduced in one study but not the other (Table 2).

\section{Monotherapy in patients who cannot tolerate statins}

A recent retrospective case-note audit has explored the response of patients to ezetimibe if they are unable to tolerate statins as part of a larger trial which also included combination therapy with ezetimibe and a statin for patients who were unable to tolerate high statin doses or failed to reach LDL-C targets with statin monotherapy. ${ }^{23}$ Ezetimibe, $10 \mathrm{mg}$, was given as monotherapy for patients $(\mathrm{n}=27)$ who had tried statins and had adverse events resulting in discontinuation of statin therapy. In this group, LDL-C was reduced by $26 \%$ and total cholesterol by $18 \%$ (both $p<0.001$ vs baseline). However, HDL-C levels were not affected and the $11 \%$ reduction in triglycerides was not

Table 2. Mean percentage change in plasma concentrations of lipid parameters from baseline to study endpoint (12 weeks treatment with ezetimibe, $10 \mathrm{mg}$, or placebo) in patients with primary hypercholestrerolaemia. ${ }^{21,22}$

\begin{tabular}{|c|c|c|c|c|}
\hline \multirow[t]{3}{*}{ Lipid parameter } & \multicolumn{4}{|c|}{ Mean change from baseline $(\%)$} \\
\hline & \multicolumn{2}{|c|}{ Dujovne et al. ${ }^{22}$} & \multicolumn{2}{|c|}{ Knopp et al. ${ }^{21}$} \\
\hline & $\begin{array}{l}\text { Placebo } \\
(n=226)\end{array}$ & $\begin{array}{l}\text { Ezetimibe } 10 \mathrm{mg} / \text { day } \\
\qquad(\mathrm{n}=666)\end{array}$ & $\begin{array}{l}\text { Placebo } \\
(n=205)\end{array}$ & $\begin{array}{l}\text { Ezetimibe } 10 \mathrm{mg} / \text { day } \\
\qquad(\mathrm{n}=622)\end{array}$ \\
\hline $\mathrm{LDL}^{\mathrm{a}} \mathrm{C}^{\mathrm{a}}$ & 0.36 & $-16.86^{*}$ & 0.79 & $-17.69^{*}$ \\
\hline Apolipoprotein B & -1.42 & $-15.52 *$ & -1.01 & $-15.38^{*}$ \\
\hline HDL-C & -1.60 & $1.31 *$ & -1.26 & $1.01 *$ \\
\hline HDL2-C & 0.55 & 1.25 & -1.14 & $5.03 *$ \\
\hline HDL3-C & 1.01 & $5.65^{*}$ & 3.94 & 2.84 \\
\hline Apolipoprotein A-I & 1.91 & 2.53 & 1.20 & 2.26 \\
\hline Total cholesterol & 0.84 & $12.48^{*}$ & 0.57 & $-12.4^{*}$ \\
\hline LDL-C:HDL-C & 2.66 & $-17.47^{*}$ & 2.27 & $-18.25^{*}$ \\
\hline TC:HDL-C & 2.99 & $-13.13^{*}$ & 2.12 & $-12.8^{*}$ \\
\hline Triglycerides & 5.74 & $-5.65^{*}$ & 2.43 & -1.71 \\
\hline Lipoprotein (a) & 16.32 & 2.83 & 1.76 & $-7.50^{*}$ \\
\hline
\end{tabular}


significant $(p>0.05)$. Mean LDL-C levels before and after starting ezetimibe monotherapy were 4.4 and $3.2 \mathrm{mmol} / \mathrm{L}$, with only $20 \%$ of patients achieving the NCEP Adult Treatment Program (ATP) III goal of $<2.6 \mathrm{mmol} / \mathrm{L}$.

\section{Ezetimibe-statin combination therapy}

As ezetimibe and statins affect distinct pathways of cholesterol biosynthesis (the exogenous and endogenous pathways, respectively), it has been hypothesised that coadministration of ezetimibe with a statin would result in a significantly greater reduction in LDL-G compared with statin monotherapy. A series of randomised, double-blind studies have compared the effect of ezetimibe in combination with statins at varying doses across their licensed dose ranges.

\section{Ezetimibe plus any statin: add-on therapy}

The clinical efficacy and safety of ezetimibe added to ongoing therapy with a variety of statins has been determined by a systematic review and meta-analysis involving five randomised controlled trials and 5039 patients. ${ }^{7}$ The included trials lasted at least 6 weeks, and patients had hypercholesterolaemia with LDL-C levels above those recommended by NCEP ATP II/III guideline criteria despite statin monotherapy, and were subsequently given add-on therapy with ezetimibe, $10 \mathrm{mg}$, or placebo. ${ }^{24-28}$ The weighted mean difference (WMD) between treatments significantly favoured the ezetimibe/ statin combination over placebo/statin for total cholesterol $(-16.1 \% ; p<0.0001)$, LDL-C $(-23.6 \% ; \quad p<0.0001)$ and HDL-C $(+1.7 \%$; $p<0.0001)$. The relative risk ( $\mathrm{RR})$ of reaching the LDL-G treatment goal was also higher for patients on ezetimibe/statin relative to those on placebo/statin (RR 3.4; $p<0.0001)$. Moreover, elevations in creatine kinase (CK) levels that were considered as adverse effects did not differ significantly between treatments. These results suggest that ezetimibe coadministered with ongoing statin therapy provides significant additional lipid-modifying effects in patients not at LDL-C goal on statin monotherapy, thus allowing more patients to reach their LDL-C goal.

In a recent open-label, single cohort study in 837 patients with hypercholesterolaemia who failed to achieve target LDL-G levels on statin monotherapy, patients were subsequently given add-on ezetimibe, $10 \mathrm{mg} .{ }^{29}$ After 6 weeks' treatment, $80.5 \%$ achieved target LDL-C levels, and LDL-C levels were reduced by an average of $30.1 \%$ across all statins and statin doses (from 3.4 $\mathrm{mmol} / \mathrm{L}$ at baseline to $2.4 \mathrm{mmol} / \mathrm{L}$ after 6 weeks' treatment).

Ezetimibe add-on therapy for patients taking statins has also been evaluated within primary care. ${ }^{27,30} \mathrm{~A}$ double-blind trial compared the addition of placebo or ezetimibe, 10 $\mathrm{mg}$, to a variety of statins for 6 weeks in a population of 3030 primary-care patients with hypercholesterolaemia, many of whom $(77 \%)$ had CHD or a CHD risk equivalent. ${ }^{27}$ The ezetimibeplus-statin combination reduced LDL-C from baseline by $25.8 \%$ compared with a reduction of $2.7 \%$ with placebo plus a statin $(p<0.001)$, and more patients reached their specific NCEP ATP III target LDL-C goal $(71.0 \%$ vs $20.6 \%$, respectively; $p<0.001)$. Drug-related clinical adverse events (ezetimibe plus statin, 5.1\%; statin monotherapy, 5.2\%) and drug-related clinical adverse events leading to treatment discontinuation (ezetimibe plus statin, 0.9\%; statin monotherapy, $1.6 \%$ ) were comparable for treatment groups. A smaller, uncontrolled openlabel study involved 130 primary care patients with hypercholesterolaemia, all with CHD or type 2 diabetes and receiving a statin, who were given ezetimibe, $10 \mathrm{mg}$, in addition to their statin therapy for 6 weeks. ${ }^{31}$ This reported similar results to the larger primary care study discussed here, with $70 \%$ of patients achieving their LDL-C goal $(<2.5 \mathrm{mmol} / \mathrm{L})$ following mean additional reductions in LDL-C of 29\% from baseline (no formal statistics were undertaken). 


\section{Ezetimibe plus any statin in statin-naïve patients}

Four, 12-week, randomised, double-blind studies with a similar trial design, and enrolling a total of nearly 2400 statin-naïve patients with primary hypercholesterolaemia, have compared the effects of ezetimibe, $10 \mathrm{mg}$, as monotherapy and in combination with atorvastatin, ${ }^{31}$ simvastatin, ${ }^{32}$ pravastatin $^{33}$ and lovastatin ${ }^{34}$ across their licensed dose ranges. Patients underwent a washout period for previous lipid-modifying drugs (including statins) of up to 12 weeks. Baseline lipid profiles were similar in these four studies (LDL-C: 3.7-6.5 $\mathrm{mmol} / \mathrm{L}$; triglycerides $\leq 4.0 \mathrm{mmol} / \mathrm{L}$ ). Significant decreases in LDL-C and triglycerides and significant increases in HDL-C were observed for the ezetimibe plus any statin group in each trial vs pooled statin groups (Figure 2).

A pooled analysis of these four trials has also been performed. ${ }^{35}$ Pooling data across all doses for all statins, the addition of ezetimibe was more effective in reducing LDL-C than statins alone $(45.2 \%$ vs $31.9 \%$, respectively), with most reductions occurring within 2 weeks and sustained for the remainder of the 12-week study period. ${ }^{35}$ With the exception of atorvastatin, 40 and 80 $\mathrm{mg}$ groups, adding ezetimibe to each statin dose led to significantly $(p<0.01)$ greater incremental mean percentage reductions in LDL-C compared with each corresponding statin monotherapy dose and also the next highest statin monotherapy dose. Coadministration of ezetimibe with the lowest statin dose resulted in similar reductions in LDL-C as the highest corresponding statin dose as monotherapy. (For example, LDL-C reductions of $51 \%$ [atorvastatin, $80 \mathrm{mg}$ ] and $50 \%$ [atorvastatin, $10 \mathrm{mg}$, plus ezetimibe, $10 \mathrm{mg}]$ ). Reductions in total cholesterol, triglycerides and Apo B, and increases in HDL-C, were also greater for combination treatment with ezetimibe plus statin than for statin monotherapy (all $p<0.01$; Figure 3). Treatment with ezetimibe plus any statin (all doses) resulted in the following changes in plasma lipid profiles: total cholesterol, $-33.4 \%$; triglycerides, $-27.3 \%$; HDL-C, $+2.9 \%$. In addition, $86 \%$ of patients given ezetimibe plus a statin met NCEP ATP III LDL-C targets compared with $68 \%$ of patients given statin monotherapy. ${ }^{35}$

\section{Combination with rosuvastatin}

A 6-week, open-label, parallel-group trial has investigated the efficacy and safety of rosuvastatin, $40 \mathrm{mg}$, given as monotherapy or in combination with ezetimibe, $10 \mathrm{mg}$, in patients $(\mathrm{n}=469)$ at high risk of cardiovascular disease (hypercholesterolaemia, history of CHD, clinical evidence of atherosclerosis or CHD risk equivalent [10-year CHD risk score $>20 \%$ ]). ${ }^{36}$ More patients receiving rosuvastatin plus ezetimibe than rosuvastatin alone achieved their ATP III LDL-C goal $(<2.6 \mathrm{mmol} / \mathrm{L} ; 94.0 \%$ vs $79.1 \%$, respectively; $p<0.001)$ and the optional LDL-C goal $(<1.8 \mathrm{mmol} / \mathrm{L})$ for very high-risk patients $(79.6 \%$ vs $35.0 \%$, respectively; $p<0.001)$. Furthermore, the combination of rosuvastatin plus ezetimibe reduced LDL-C more than rosuvastatin monotherapy $(69.8 \%$ vs $57.1 \%$, respectively; $p<0.001)$. Other components of the lipid/lipoprotein profile were also significantly $(p<0.001)$ improved with rosuvastatin plus ezetimibe, and both treatments generally were well tolerated. The percentage of patients who discontinued treatment as a result of any adverse event was $1.3 \%$ and $2.5 \%$ in the rosuvastatin monotherapy and the rosuvastatinplus- ezetimibe combination therapy groups, respectively. Treatment-related adverse events were the reason for discontinuation in 3 patients $(1.3 \%)$ in the monotherapy group and 2 patients $(0.8 \%)$ in the combination therapy group.

\section{Combination with atorvastatin}

Ezetimibe coadministered with atorvastatin has been investigated in a long-term clinical study, ${ }^{37}$ in patients with $\mathrm{CHD}^{26,38}$ and for achieving LDL-G goals in very high-risk patients. ${ }^{39}$ The long-term clinical study consisted of a 12-month extension of a 12-week, double-blind, placebo-controlled trial comparing ezetimibe 
Figure 2. Mean percentage change from baseline in low-density lipoprotein cholesterol (LDL-C), high-density lipoprotein cholesterol (HDL-C) and triglycerides in patients with primary hypercholesterolaemia treated for 12 weeks with ezetimibe monotherapy, statin monotherapy or ezetimibe-statin combination therapy.
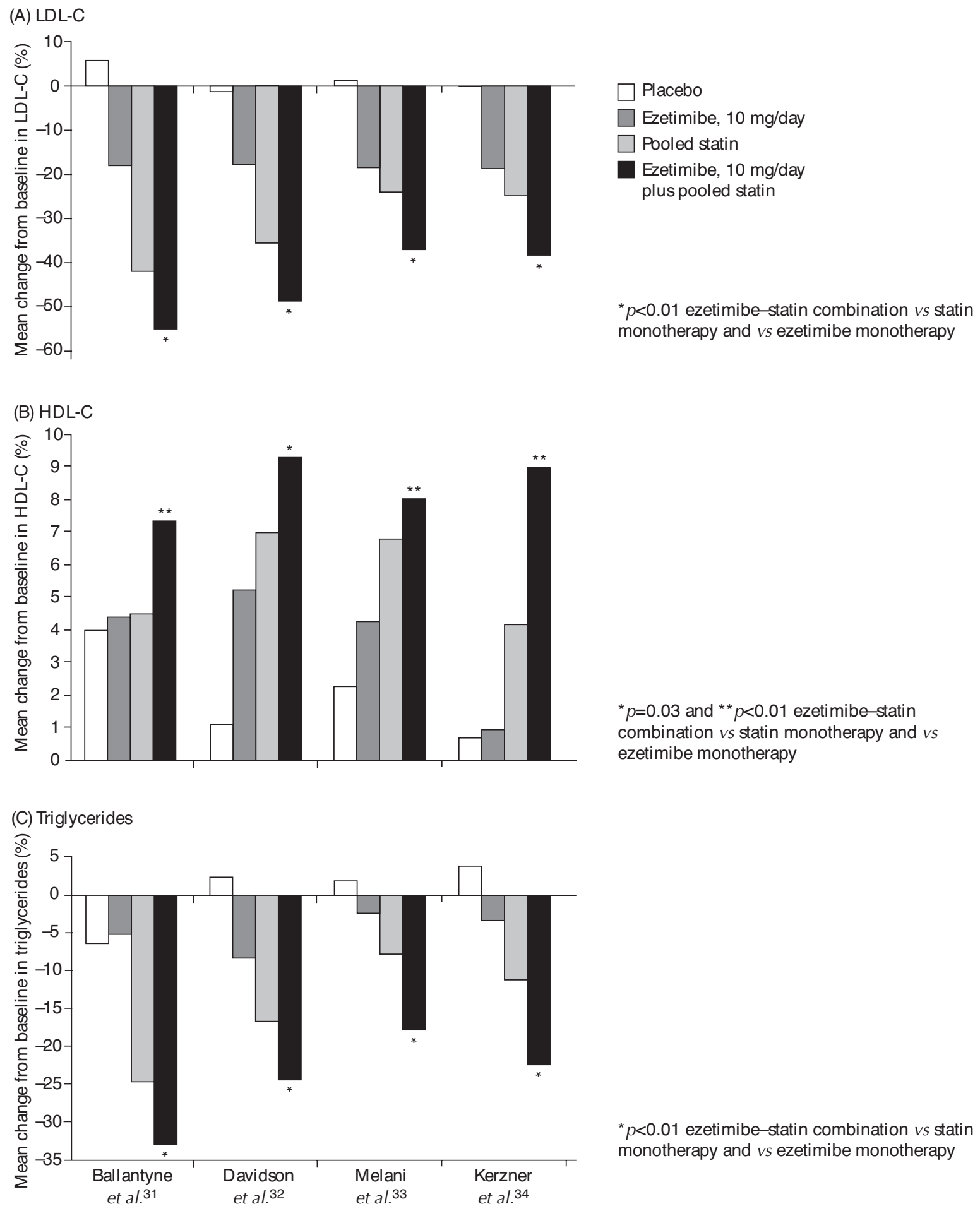

${ }^{*} p<0.01$ ezetimibe-statin combination vs statin monotherapy and vs ezetimibe monotherapy 
Figure 3. Percentage change from baseline in lipids and lipoproteins following 12 weeks of treatment with statin alone or statin plus ezetimibe, $10 \mathrm{mg} /$ day, based on a pooled analysis of four trials. ${ }^{35}$

(A) Direct LDL-C

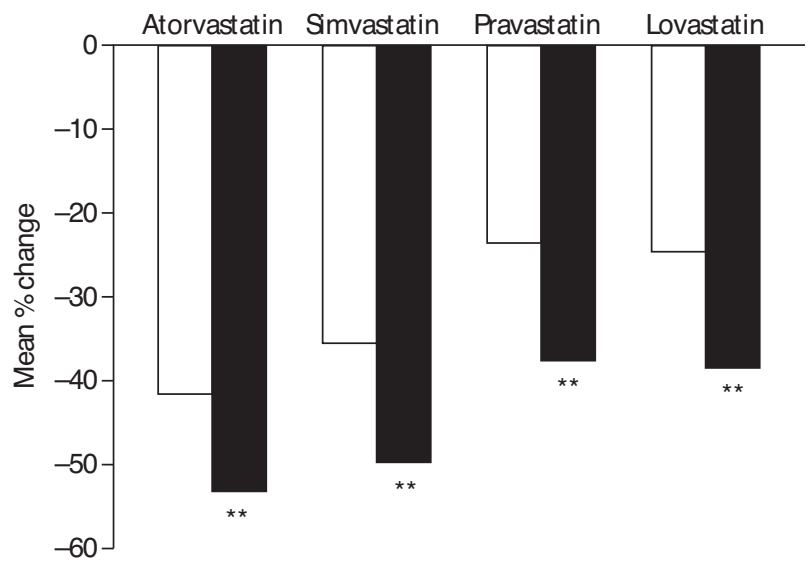

(C) HDL-C

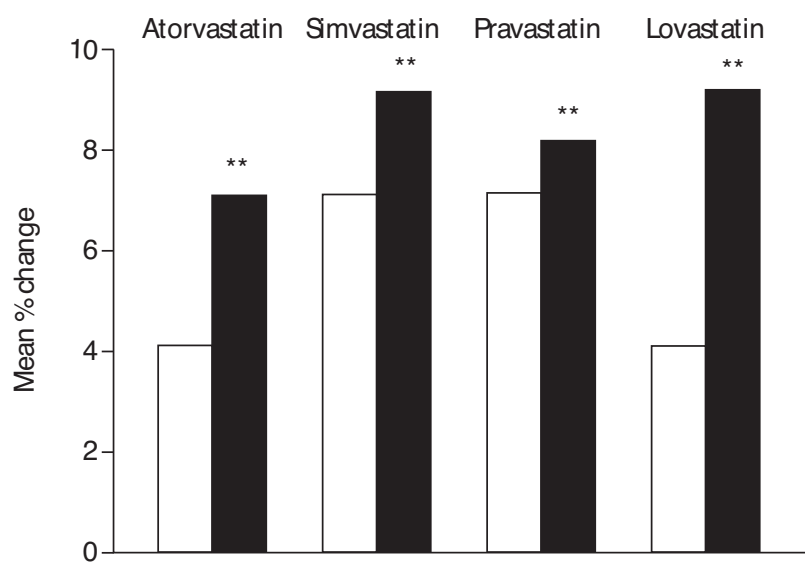

(E) TG

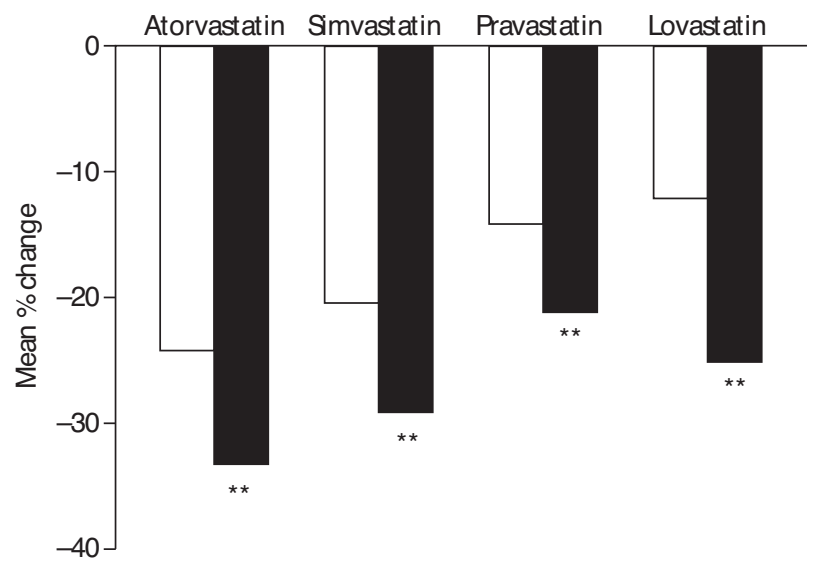

(B) Calculated LDL-C

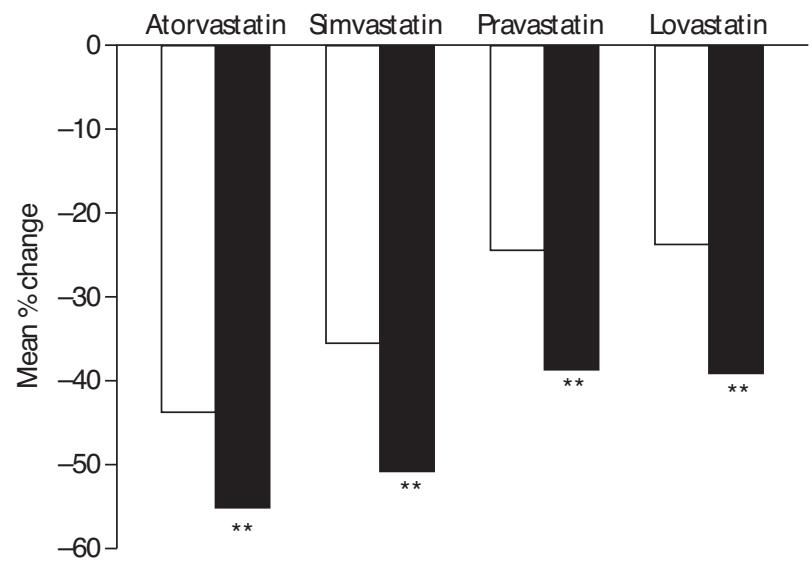

(D) TC

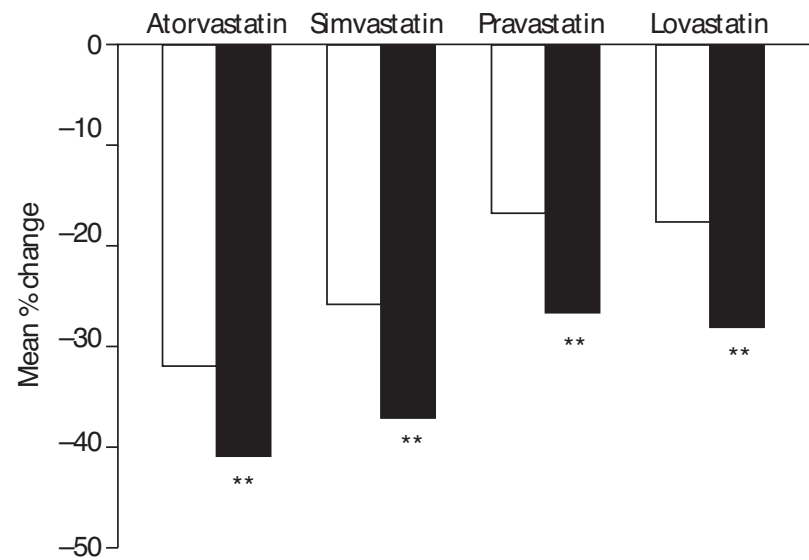

(F) Apo B

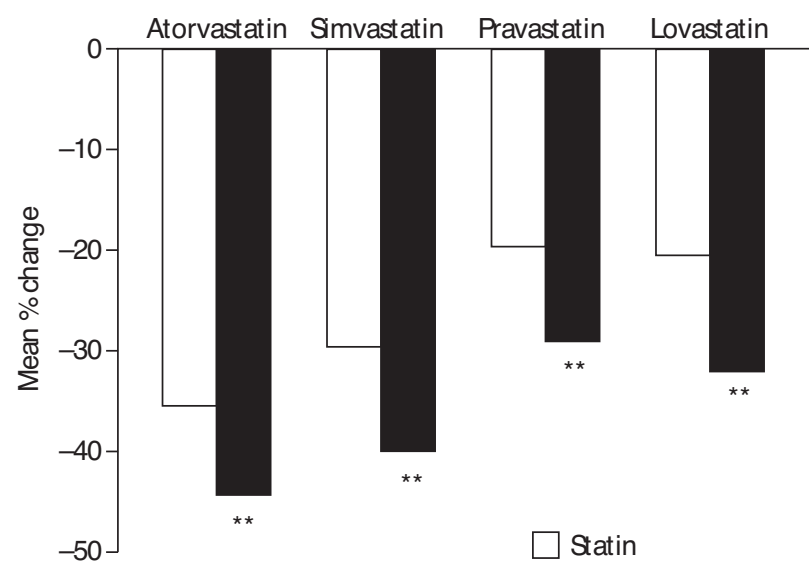

Apo B, apolipoprotein B; HDL-C, high-density lipoprotein cholesterol; LDL-C, low-density lipoprotein cholesterol; TC, total cholesterol; TG, triglycerides.

${ }^{* *}, p<0.01$. 
and atorvastatin, with both drugs given as monotherapies and in combination. ${ }^{31,37}$ In the extension study, 246 patients were randomised in a double-blind manner to ezetimibe, $10 \mathrm{mg}$, or matching placebo, with both groups given open-label atorvastatin, $10 \mathrm{mg}$. At intervals of 6 weeks, patients not achieving NGEP ATP II LDL-G goals were titrated to the next higher dose of atorvastatin. More patients receiving ezetimibe plus atorvastatin achieved the LDL-G goal than those given atorvastatin monotherapy $(91 \%$ vs $78 \%$, respectively; $p=0.02)$. After 6 weeks, combination treatment produced greater reductions in LDL-C $(53 \%$ os $37 \%$ ), total cholesterol $(38.8 \%$ vs $26.0 \%)$ and triglycerides $(28 \%$ vs $12 \%)$ and similar increases in HDL-C (4.6\% vs 4.5\%) compared to atorvastatin monotherapy, respectively, and these changes were maintained and significant at 1 year $(p<0.01$ for LDL-C, total cholesterol and triglycerides). Furthermore, ezetimibe plus atorvastatin and atorvastatin monotherapy groups were similar with regard to incidence of all adverse events $(71 \%$ vs $67 \%)$, treatmentrelated adverse events $(22 \%$ vs $27 \%)$ and discontinuations because of either all adverse events $(9 \%$ vs $7 \%)$ or treatment-related adverse events $(6 \%$ vs $7 \%)$.

Patients with CHD, or CHD-risk equivalents, have been given atorvastatin as monotherapy or in combination with ezetimibe in two randomised, double-blind studies. ${ }^{26,38}$ Treatment-naïve patients $(\mathrm{n}=148)$ with baseline LDL-C levels between 3.3 and $5.2 \mathrm{mmol} / \mathrm{L}$ were given 6-weeks' open-label treatment with atorvastatin, $10 \mathrm{mg}$, plus double-blind treatment with either ezetimibe, $10 \mathrm{mg}$, or placebo. ${ }^{38}$ Reductions from baseline LDL-C levels were greater for ezetimibe plus atorvastatin than with atorvastatin monotherapy $(50.5 \%$ vs $36.5 \%$, respectively; $p<0.0001)$. Moreover, the new Joint British Societies (JBS 2) LDL-C goal of $<2 \mathrm{mmol} / \mathrm{L}$ was reached by $62 \%$ of the ezetimibe-plus-atorvastatin group and only
$12 \%$ of the atorvastatin group $(p<0.0001)$. The JBS-2 LDL-C minimal treatment standard $(<3.0 \mathrm{mmol} / \mathrm{L})$ was reached by $93 \%$ of the ezetimibe plus atorvastatin group and $79 \%$ of the atorvastatin group $(p<0.0001)$. Overall, these treatment-naïve patients with $\mathrm{CHD}$ or CHD risk equivalent were 12-times more likely to reach LDL-C targets with ezetimibe plus atorvastatin than atorvastatin alone (odds ratio 12.1 ; 95\% confidence interval 5.8-25.1; $p<0.0001)$. A larger trial has been conducted in patients with documented CHD who had failed to reach the NCEP ATP III LDL-C goal of $\leq 2.60 \mathrm{mmol} / \mathrm{L}(\mathrm{n}=450)$ despite at least 6 weeks' treatment with atorvastatin, 10 or 20 mg/day. ${ }^{26}$ Patients continued open-label treatment with atorvastatin, 10 or $20 \mathrm{mg}$, and were randomised, in a double-blind manner, to receive ezetimibe, $10 \mathrm{mg}$, or placebo, for 6 weeks. Coadministration of ezetimibe and atorvastatin led to greater reductions in LDL-C, total cholesterol and triglycerides, and greater increases in HDL-C than those given atorvastatin monotherapy ( $p \leq 0.001$ for all comparisons). More patients achieved the LDL-C goal of (2.6 mmol/L) with ezetimibe and atorvastatin than with atorvastatin alone $(81.3 \%$ vs $21.8 \%$, respectively; $p \leq 0.001)$. Interestingly, the dose of atorvastatin made little difference to reaching this target. For the atorvastatin, $10 \mathrm{mg}$ group, $81 \%$ given combination treatment and 24\% given atorvastatin monotherapy reached the LDL-C target, and in the atorvastatin, $20 \mathrm{mg}$ group, 82\% given combination treatment and $19 \%$ given atorvastatin monotherapy reached the LDL-C target (Figure 4). ${ }^{26}$

Patients $(n=621)$ with heterozygous familial hypercholesterolaemia, CHD or at least two cardiovascular risk factors, and an LDL-G level of at least $3.37 \mathrm{mmol} / \mathrm{L}$, despite treatment with atorvastatin, $10 \mathrm{mg}$, for at least 6 weeks, continued with open-label atorvastatin, $10 \mathrm{mg}$, and were randomised in a double-blind manner to receive ezetimibe, $10 \mathrm{mg}$, or an additional 
Figure 4. Percentage of patients with $\mathrm{CHD}$ achieving the LDL-C goal of $\leq 2.60 \mathrm{mmol} / \mathrm{L}$ after 6 weeks' treatment with atorvastatin, 10 or $20 \mathrm{mg}$, with or without ezetimibe, $10 \mathrm{mg}$, and stratified by atorvastatin dose. ${ }^{26}$

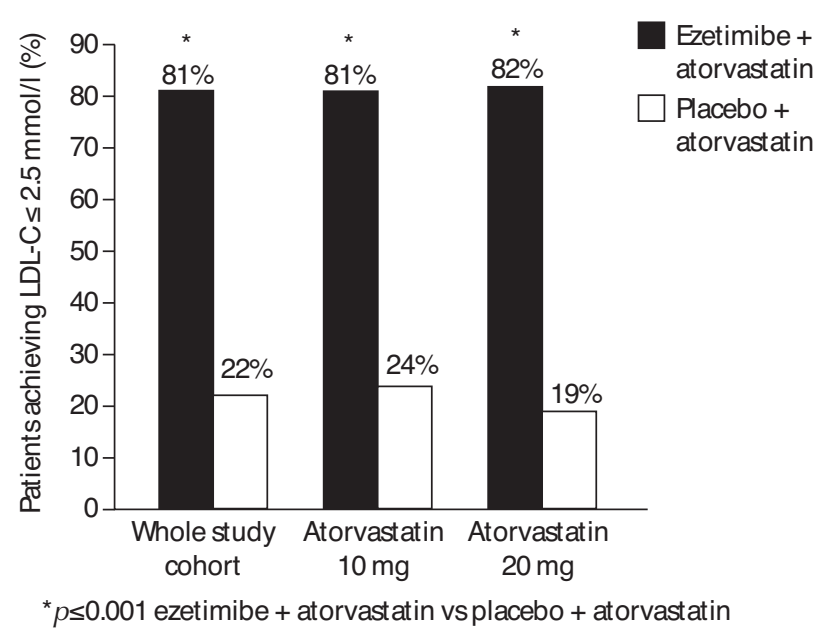

$10 \mathrm{mg}$ of atorvastatin for 14 weeks' treatment. ${ }^{39}$ The atorvastatin dose was doubled in both groups at regular intervals, when required, to achieve LDL-C target of $2.59 \mathrm{mmol} / \mathrm{L}$, to a maximum of $40 \mathrm{mg}$ (combination group) and $80 \mathrm{mg}$ (atorvastatin monotherapy group). Combination therapy provided a more effective way of reducing LDL-C levels in patients at high risk for $\mathrm{CHD}$ or with $\mathrm{CHD}$ : the LDL-C goal of $2.59 \mathrm{mmol} / \mathrm{L}$ was reached by $22 \%$ in the combination therapy group (a reduction of $33.2 \%$ ) and $7 \%$ (a reduction of $20.3 \%$ ) in the atorvastatin group $(p<0.01$ for groups reaching LDL-C goal).

\section{Ezetimibe-fibrate combination therapy}

Some patients suffer from mixed dyslipidaemia, characterised by hypercholesterolaemia and hypertriglyceridaemia. More specifically, these patients have high LDL-C and triglyceride levels, reduced HDL-G levels and a preponderance of small, dense LDL particles. This highly atherogenic 'lipid triad' is often associated with type 2 diabetes or the metabolic syndrome. ${ }^{40}$ Though statins are excellent at reducing LDL-C levels, they are less effective than fibrates at increasing HDL-C and decreasing triglyceride levels, whilst fibrates have only a moderate LDL-C-lowering effect. Thus, statins and fibrates have complementary actions and are often used together for mixed dyslipidaemia, though there have been safety concerns with statinfibrate combinations (particularly with gemfibrozil) and a proportion of patients are also intolerant to statins. One alternative is to use an ezetimibefibrate combination.

Two noteworthy trials have investigated the efficacy and safety of ezetimibe and a fibrate in patients with mixed hyperlipidaemia. ${ }^{41,42} \mathrm{~A}$ total of 625 patients with mixed dyslipidaemia (LDL-C, 3.4-5.7 mmol/L [2.6-4.7 mmol/L for those with type 2 diabetes]; triglycerides, 2.3$5.7 \mathrm{mmol} / \mathrm{L}$; and no history of CHD or CHDequivalent disease [except type 2 diabetes], or a 10-year CHD risk of less than 20\%]) were randomised to one of four daily treatments for 12 weeks: ezetimibe, $10 \mathrm{mg}$; fenofibrate, $160 \mathrm{mg}$; ezetimibe, $10 \mathrm{mg}$, plus fenofibrate, $160 \mathrm{mg}$; or placebo. ${ }^{41}$ Coadministration of ezetimibe and fenofibrate reduced LDL-C by $20.4 \%$, triglycerides by $44.0 \%$, and increased HDL-C by $19.0 \%$. Of these parameters, however, only LDL-C levels were affected to a significantly greater extent than with fenofibrate monotherapy, which reduced LDL-C by $13.4 \%$ ( $p<0.001$ vs combination treatment). All treatments were well tolerated, with a similar frequency of adverse events in each group, and there were no cases of myopathy or CK elevations greater than 10-times the upper limit of normal (ULN). Patients from this base study continued into a further 48-week, double-blind extension trial unless there was a condition that would interfere with their ability to participate. ${ }^{42}$ All participating patients $(n=576)$ continued with their base treatment except those in the ezetimibe monotherapy group (switched to ezetimibe plus fenofibrate) and the placebo group (switched to fenofibrate monotherapy). Treatment with ezetimibe plus 
fenofibrate produced greater reductions in LDL-C than fenofibrate monotherapy $(22 \%$ vs $9 \%$, respectively; $p<0.001)$. There were also greater improvements in triglycerides, HDL-C, total cholesterol and Apo-B with combination treatment vs fibrate monotherapy (Table 3$).{ }^{42}$ It should be noted, however, that the mean period of drug exposure was lower in the fenofibrate than the combination therapy group (212 days vs 271 days, respectively). This was because of the number of discontinuations owing to lack of efficacy, defined as LDL-G >0.39 mmol/L above each patient's NCEP ATP III risk-specific goal at week 12 or later during the extension, which resulted in $50.8 \%$ in the fenofibrate group and $24.1 \%$ in the combination group discontinuing. Both treatments had a good safety and tolerability profile, with similar proportions of patients in the ezetimibe and fenofibrate and fenofibrate monotherapy groups with adverse events $(67.4 \%$ vs $61.4 \%$, respectively) and treatment-related adverse events (13.8\% vs $16.1 \%$, respectively). Furthermore, the proportion of patients with $\mathrm{CK}$ elevations of at least three-times the ULN was similar in the combination treatment and fibrate monotherapy groups $(1.2 \%$ vs $1.7 \%$, respectively), and there were no cases of myopathy or $\mathrm{CK}$ elevations greater than 10-times the ULN.

Table 3. Percentage change from baseline ${ }^{a}$ in lipids and lipoproteins, for patients with mixed dyslipidaemia, after up to 48 weeks of treatment with ezetimibe, $10 \mathrm{mg} /$ day, plus fenofibrate, $160 \mathrm{mg} /$ day, or fenofibrate monotherapy, $160 \mathrm{mg} /$ day. ${ }^{42}$

\begin{tabular}{lccc}
\hline & $\begin{array}{c}\text { Ezetimibe plus } \\
\text { fenofibrate }\end{array}$ & $\begin{array}{c}\text { Fenofibrate } \\
\text { monotherapy }\end{array}$ & p-value \\
\hline LDL-C & -22.0 & -8.6 & $<0.001$ \\
Triglycerides & -46.0 & -41.8 & 0.002 \\
HDL-C & 20.9 & 17.8 & 0.02 \\
Total cholesterol & -23.2 & -13.6 & $<0.001$ \\
Apo-B & -25.2 & -16.2 & $<0.001$ \\
\hline
\end{tabular}

aBaseline values were recorded at the start of the preceding 12-week base study. ${ }^{41}$

Apo B, apolipoprotein B; HDL-C, high-density lipoprotein cholesterol; LDL-C, low-density lipoprotein cholesterol.

\section{Ezetimibe-statin-fibrate combination therapy}

The efficacy of a combination of ezetimibe with both a statin and a fibrate in patients with mixed hyperlipidaemia has been investigated in a randomised, double-blind trial. ${ }^{43}$

The study involved 611 patients with no history of coronary heart disease and with LDL-C levels of $3.4-5.7 \mathrm{mmol} / \mathrm{L} \quad(2.6-4.7 \mathrm{mmol} / \mathrm{L}$ for type 2 diabetics) and triglyceride levels of 1.7-5.7 mmol/L. Patients were randomised in a 3:3:3:1 ratio to receive either ezetimibe/ simvastatin, 10/20mg (combination tablet), plus fenofibrate, 160mg; or ezetimibe/simvastatin, $10 / 20 \mathrm{mg}$; or fenofibrate, 160mg; or placebo. After 12 weeks' treatment the reduction in LDL-C level was significantly $(p<0.05)$ greater in the ezetimibe/simvastatin plus fenofibrate group than in the fenofibrate or placebo groups $(45.8 \%$ vs $15.7 \%$ vs $3.5 \%$ ), but not compared with the ezetimibe/simvastatin group (47.1\%). Increases in HDL-C levels were significantly $(p<0.05)$ greater in the ezetimibe/simvastatin plus fenofibrate group than in the ezetimibe/ simvastatin or placebo groups $(18.7 \%$ vs $9.3 \%$ vs $1.1 \%$, but not when compared with fenofibrate $(18.2 \%)$. A similar pattern was seen for ApoA-I. Triglycerides, non-HDL-C and Apo-B levels were all reduced to a significantly greater extent $(p<0.05)$ in the ezetimibe/simvastatin plus fenofibrate group than in any of the other groups. Therefore, overall, the combination of ezetimibe/ simvastatin plus fenofibrate produced complementary improvements in the lipid profile in patients with mixed hyperlipidaemia. In addition, the tolerability profile of the triple combination therapy was similar to that of the individual components.

\section{Ezetimibe-bile acid-binding resin combination therapy}

Bile acid-binding resins enhance cholesterol excretion by inhibiting bile acid reabsorption and 
increasing cholesterol conversion into bile acids. ${ }^{44}$ The use of newer resins such as colesevelam (which is more specific for LDL-C than older resins and so can be used at much lower doses) has been evaluated in combination with ezetimibe in a number of studies. However, these are mostly pilot studies: 20 and 12 patients were randomised in two studies, thus it is impossible to draw firm conclusions. However, the results from one study suggest that colesevelam does not have a significant additive cholesterol-lowering benefit in combination with ezetimibe, ${ }^{43}$ whilst another (in patients who were intolerant to, or refused, statin treatment) showed a significant additive effect, with LDL-C reduced by an additional 20\% over either treatment as monotherapy $(p<0.005) .{ }^{45}$ A slightly larger study has been conducted in 40 patients, in the form of a prospective chart review in which ezetimibe, $10 \mathrm{mg}$, was added to a stable regimen which included a variety of bile acid-binding resins (colesevelam, colestipol or cholestyramine), additional statins $(\mathrm{n}=32)$, and extended-release niacin $(n=19) .{ }^{46}$ The addition of ezetimibe to these (already complex) regimens resulted in additional improvements in lipid and lipoprotein profiles (LDL-C, $-19 \%$; triglycerides, $-14 \%$; total cholesterol, $-18 \%$; all $p \leq 0.03)$, but it is difficult to comment on the significance of these results due to the heterogeneity of the patient population and treatment regimens, low patient numbers, the prospective nature of the study and the lack of a control group. A prospective, double-blind, parallel-group study has compared 6 weeks of treatment with ezetimibe, $10 \mathrm{mg}$, plus colesevelam with ezetimibe monotherapy in 86 patients. ${ }^{47}$ This showed significant reductions in LDL-C for combination treatment compared with ezetimibe monotherapy $(32.3 \%$ vs $21.4 \%$, respectively; $p<0.0001$ ), but no significant difference in triglyceride levels. A larger study is still required to assess the utility of bile acidbinding resins in combination with ezetimibe, though this treatment option may be useful for some patients who cannot tolerate statins.

\section{Ezetimibe and plant sterols}

The American Heart Association (AHA) recommends a diet containing $2 \mathrm{~g} /$ day plant stanols/sterols to lower LDL-C. ${ }^{48}$ In addition to inhibiting cholesterol absorption, ezetimibe inhibits the absorption of plant sterols. ${ }^{49,50}$ The combination of ezetimibe, $10 \mathrm{mg} /$ day, with a diet containing $2 \mathrm{~g} /$ day plant sterols did not produce a further significant reduction in LDL-C compared with ezetimibe alone (decreases in LDL-C of $1.06 \mathrm{mmol} / \mathrm{L}$ and $0.94 \mathrm{mmol} / \mathrm{L}$, respectively) in 40 patients with mild hypercholesterolaemia (baseline LDL-C $4.5 \mathrm{mmol} / \mathrm{L}$ ) after 4 weeks in a double-blind, placebo-controlled, open-label, crossover trial. ${ }^{51}$

\section{Familial hypercholesterolaemia}

Patients with familial hypercholesterolaemia $(\mathrm{FH})$ have defects in one or both copies of the gene that encodes the LDL-C receptor (heterozygous and homozygous $\mathrm{FH}$, respectively), and so have excessively elevated LDL-C levels, placing them at very high risk of cardiovascular events. Thus, aggressive lipid-modifying therapy is needed in this group, with LDL-C lowering the over-riding priority.

Patients undergoing LDL apheresis therapy and receiving statin therapy $(95 \%$ of patients taking the maximum tolerated statin dose), and with severe hypercholesterolaemia, without $(n=18)$ or with $(n=2)$ CHD, were given ezetimibe, $10 \mathrm{mg}$, for 5 weeks in a randomised, double-blind, crossover trial. ${ }^{52}$ Pre-apheresis LDL-C levels fell from 4.1 to $3.4 \mathrm{mmol} / \mathrm{L}$ with ezetimibe treatment, a reduction of $16 \%(p<0.0001)$, and post-apheresis LDL-G levels also fell (from 1.3 to $1.1 \mathrm{mmol} / \mathrm{L}$ ) with ezetimibe treatment, a reduction of $14 \%$ $(p<0.05)$. These further, clinically significant decreases in LDL-C levels with additional ezetimibe treatment were independent of statin dose or dietary behaviour. Although patients with homozygous $\mathrm{FH}$ were excluded from this trial, a small open-label study of add-on therapy 
with ezetimibe, $10 \mathrm{mg}$, for 12 weeks, has been performed in six patients with homozygous $\mathrm{FH}$ and undergoing apheresis and taking atorvastatin or simvastatin. ${ }^{53}$ LDL-C levels were reduced by an average of $9.0 \%$ during ezetimibe treatment ( $p$-value not given), and though this effect is not very spectacular, ezetimibe may still be a useful adjunct therapy in this difficult-to-treat population.

Patients with homozygous $\mathrm{FH}(\mathrm{n}=50)$, taking atorvastatin or simvastatin (both $40 \mathrm{mg} /$ day) at baseline, with or without apheresis, were randomised in a double-blind manner to receive 12 weeks of treatment with either ezetimibe, 10 mg, plus atorvastatin or simvastatin (both 40 $\mathrm{mg}$ /day), ezetimibe, $10 \mathrm{mg}$, plus atorvastatin or simvastatin (both $80 \mathrm{mg} /$ day), or monotherapy with atorvastatin or simvastatin (both $80 \mathrm{mg}$ / day). ${ }^{54}$ This study showed that the ezetimibestatin, $80 \mathrm{mg} /$ day, combination therapy, in particular, was very effective, having four-times the LDL-C-lowering potency as a doubling of the statin dose from 40 to $80 \mathrm{mg}(-27.5 \%$ vs $-7.0 \%$, respectively; $p=0.0001$ ).

A much larger, prospective study has investigated the use of ezetimibe in 200 patients with $\mathrm{FH}$ who were defined as statin resistant (predicted LDL-C response of $<90 \%$ of the predicted response) or statin intolerant (inability to tolerate any statin therapy if the patient had tried two or more statins), with all patients having LDL-C levels greater than $3.0 \mathrm{mmol} / \mathrm{L} .{ }^{55}$ All patients were stabilised using the American Heart Association (AHA) Step 2 diet and were encouraged to eat stanol products, particularly the $22 \%$ of patients who were not taking statins due to statin intolerance. Ezetimibe monotherapy resulted in a 7\% reduction in LDL-C, ezetimibestatin therapy reduced LDL-C by $11 \%$, and the overall the proportion of patients reaching the $3.0 \mathrm{mmol} / \mathrm{L}$ LDL-C target increased from 5.5\% to $18 \%$ (no $p$-values given).

\section{Efficacy in special populations}

Ezetimibe has been used in several special populations, including 191 patients with type 2 diabetes and 195 with the metabolic syndrome, given in combination with ongoing statin therapy, as documented in a post-hoc analysis of a larger randomised, double-blind, placebo-controlled trial. ${ }^{55}$ Ezetimibe-plus-statin combination therapy was more effective than statin monotherapy for reducing $\mathrm{LDL}-\mathrm{C}$, triglycerides, total cholesterol and Apo-B levels (all $p<0.001$ ), and treatment effects were consistent regardless of the presence or absence of diabetes or metabolic syndrome. Another post-hoc analysis that investigated the relative lipid-modifying effects of ezetimibe as an add-on therapy to statin treatment found that it was equally effective and well tolerated in the elderly (mean age 69 years; $n=579$ ) and the very elderly (mean age 79 years; $n=466) .{ }^{57}$ Furthermore, a pooled analysis of four, randomised, double-blind trials which compared statin monotherapy, ezetimibe plus a statin, or placebo, examined whether there were any differences in efficacy or safety for patients with primary hypercholesterolaemia $(n=1861)$ for the ranges: age less than 65 years vs at least 65 years, and age less than 75 years $v s$ at least 75 years. ${ }^{58}$ The additional improvements in lipid profiles demonstrated with combination therapy vs statin monotherapy were generally independent of age. For example, combination therapy resulted in an additional LDL-G reduction of $12.8 \%$ (<65 years), $15.5 \%$ ( $\geq 65$ years), $13.5 \% \quad(<75$ years) and $14.5 \%$ ( $\geq 75$ years). Moreover, there was a similar incidence of adverse events and muscle-related adverse events in each age group compared with statin monotherapy. Thus combination treatment with ezetimibe plus a statin was associated with a favourable efficacy and safety profile across these age groups. In addition to the main special population groups of patients with diabetes and the elderly, ezetimibe 
has also demonstrated efficacy in smaller special populations. These include patients with HIV, with ezetimibe, $10 \mathrm{mg}$, given as monotherapy (LDL-C reduced by $20 \%$ in 20 patients), ${ }^{59}$ in combination with pravastatin, $20 \mathrm{mg} /$ day, in patients $(n=19)$ with a poor response to statins, ${ }^{60}$ and in 33 patients as add-on therapy to maximum tolerated doses of statins (LDLC reductions of $35 \%$ compared with statin monotherapy; $p<0.001) .{ }^{61}$ Ezetimibe has also demonstrated efficacy in renal transplant patients in a retrospective analysis of 34 patients as monotherapy in patients $(n=8)$ who had failed statin therapy (LDL-C was reduced significantly [figure not given]; $p=0.036$ ) and LDL-C was reduced in combination with statins $(n=25)$ or colesevelam $(n=1)$, with reductions in LDL-C, triglycerides, total cholesterol and increases in HDL-C (all $p<0.05) .{ }^{62}$ A prospective trial of ezetimibe, $10 \mathrm{mg}$, as add-on treatment to highdose statin treatment in renal transplant patients $(\mathrm{n}=18)$ also showed significant reductions in LDL-C levels $(p<0.005) .{ }^{63}$ A small group of cardiac transplant patients $(n=16)$ unable to reach target lipid levels with statin treatment also benefited from add-on treatment with ezetimibe, $10 \mathrm{mg}$, with significant reductions in total cholesterol and LDL-G levels $(p=0.003$ and $p<0.001$, respectively). ${ }^{64}$

\section{SAFETY AND TOLERABILITY}

The safety and tolerability of ezetimibe as monotherapy and in combination with other lipid-modifying therapies has been discussed briefly throughout this review, and in general, the incidence of adverse effects is comparable to patients receiving placebo or statin monotherapy. ${ }^{21,22,31-34,65,66}$ Adverse effects in these patients receiving ezetimibe were usually of a mild, transient and non-specific nature, and included headaches and gastrointestinal disorders, with a similar discontinuation rate due to adverse events in ezetimibe- and placebotreated patients.

\section{PHARMACOECONOMICS}

The cost-effectiveness of coadministering ezetimibe with statin-treated patients who have failed to reach their lipid goal has been assessed using a decision-analytic model applied to Germany, Spain and Norway. ${ }^{67}$

This study found that for patients with CHD, the estimated incremental cost-effectiveness per life-year gained was under $€ 18,000$ for ezetimibe coadministration and $€ 26,000$ compared with strategies based on observed statin titration rates and a 'titrate to goal' statin therapy. For non-CHD patients the costs for ezetimibe coadministration and statin titration strategies were $€ 26,000$ and $€ 48,000$, respectively. A second pharmacoeconomic study for Canadian patients investigated the addition of ezetimibe to ongoing atorvastatin therapy using a Markov model. ${ }^{68}$ This study found that the costeffectiveness ratios of add-on ezetimibe therapy and either fixed-dose atorvastatin or atorvastatin titration favoured the adoption of ezetimibe within the Canadian healthcare system.

The use of ezetimibe for hypercholesterolaemia has recently been appraised by the National Institute for Health and Clinical Excellence (NICE) in England and Wales. ${ }^{69}$ The costeffectiveness of ezetimibe was modelled in five different scenarios: ezetimibe plus current statin versus current statin titrated to next dose; ezetimibe monotherapy versus no treatment in patients where statins are contraindicated or not tolerated; ezetimibe plus non-proprietary simvastatin versus atorvastatin; ezetimibe plus current statin versus current statin alone; ezetimibe plus rosuvastatin versus rosuvastatin alone. The analysis took a UK National Health Service (NHS) perspective, assumed a baseline LDL-G of $3.5 \mathrm{mmol} / \mathrm{L}$, and a lifetime horizon. 
The incremental cost per quality adjusted lifeyear (QALY) was $£$ 15-4600 for ezetimibe plus simvastatin versus atorvastatin independent of age, sex or prior $\mathrm{CV}$ disease and NICE recommended that adding ezetimibe to initial statin therapy is a cost-effective use of NHS resources compared with switching to another statin. Furthermore, from the clinical perspective, adding ezetimibe to initial statin therapy titrated to the optimum dose was concluded to be a useful alternative to switching to another statin. Ezetimibe plus appropriately titrated initial statin should therefore be considered instead of switching statins.

In patients aged 45 to 65 years, the incremental cost per QALY of ezetimibe with current statin versus current statin titrated to the next dose was between $£ 24,000$ and $£ 31,000$. Also in this age group, the incremental cost per QALY for ezetimibe as monotherapy was £24-30,000, which is within the $\AA^{20-30,000}$ threshold judged to be cost effective. Moreover, although the incremental cost for monotherapy was higher in patients aged 75 years (£33-42,000 per QALY gained), ezetimibe monotherapy was recommended for the treatment of all adults with hypercholesterolaemia in whom statins are contraindicated or poorly tolerated, since there are few other therapeutic options. The incremental cost per QALY for ezetimibe was $\AA_{19-24,000}$ when coadministered with current statin or rosuvastatin versus current statin or rosuvastatin alone, in patients aged 45-65 years in whom cholesterol targets were not achieved. NICE also concluded that ezetimibe should be prescribed based on the lowest acquisition cost, when the decision has been made to give it with a statin.

\section{SUMMARY POINTS}

- Ezetimibe acts to inhibit the absorption of dietary and biliary cholesterol across the intestinal wall, and is given as a once-daily $10 \mathrm{mg}$ tablet, either as monotherapy or in combination with other lipid-modifying agents.

- Given as monotherapy, ezetimibe significantly reduces LDL-C, total cholesterol and triglycerides, and increases HDL-C, with LDL-C levels reduced by about $17 \%$ from baseline. This can be particularly useful for patients who are statin intolerant.

- Ezetimibe can be used as an add-on therapy to statin treatment, and further modifies patients' lipid profiles, increasing the likelihood of reaching LDL-C goals. This is useful in patients who are unable to tolerate a high dose of statin, in patients who cannot reach lipid targets despite high statin doses, or as an alternative to statin titration.

- The combination of ezetimibe plus a fibrate may be particularly advantageous in patients with mixed dyslipidaemia, and when given in combination with apheresis (with or without a statin), ezetimibe can further reduce LDL-C levels for patients with familial hypercholesterolaemia.

- The safety and tolerability profile of ezetimibe has been assessed thoroughly as monotherapy and in combination with statins, and is comparable to patients receiving placebo alone or statin monotherapy, respectively.

- NICE recommends that adding ezetimibe to initial statin therapy is cost-effective compared with switching statins. 


\section{REFERENCES}

1 Grundy SM, Gleeman JI, Merz CN et al. Implications of recent clinical trials for the National Cholesterol Education Program Adult Treatment Panel III Guidelines. 7 Am Coll Cardiol 2004; 44: 720-32.

2 British Heart Foundation (BHF). Coronary Heart Disease Statistics 2007. Available from www.heartstats.org.

3 MRC/BHF Heart Protection Study of cholesterol lowering with simvastatin in 20,536 high-risk individuals: a randomised placebo-controlled trial. Lancet 2002; 360: 7-22.

4 Randomised trial of cholesterol lowering in 4444 patients with coronary heart disease: the Scandinavian Simvastatin Survival Study (4S). Lancet 1994; 344: 1383-9.

5 Pearson TA, Laurora I, Chu H, Kafonek S. The lipid treatment assessment project (L-TAP): a multicenter survey to evaluate the percentages of dyslipidemic patients receiving lipid-lowering therapy and achieving low-density lipoprotein cholesterol goals. Arch Intern Med 2000; 160: 459-67.

6 Third Report of the National Cholesterol Education Program (NCEP) Expert Panel on Detection, Evaluation, and Treatment of High Blood Cholesterol in Adults (Adult Treatment Panel III) final report. Circulation 2002; 106: 3143-421.

7 Mikhailidis DP, Sibbring GC, Ballantyne CM, Davies GM, Catapano AL. Meta-analysis of the cholesterol-lowering effect of ezetimibe added to ongoing statin therapy. Curr Med Res Opin 2007; 23: 2009-26.

8 Mikhailidis DP, Wierzbicki AS, Daskalopoulou SS et al. The use of ezetimibe in achieving low density lipoprotein lowering goals in clinical practice: position statement of a United Kingdom consensus panel. Curr Med Res Opin 2005; 21: 959-69.

9 Chambers SR. Drug review - ezetimibe. Drugs in Context 2003; 1: 15-32.

10 Chrisp P. Inegy - effective combination treatment to target LDL - C levels. Drugs in Context 2008; 4: 39 -56.

11 Rosenblum SB, Huynh T, Afonso A et al. Discovery of 1-(4-fluorophenyl)-(3R)-[3-(4-fluorophenyl)-(3S)hydroxypropyl]-(4S)-(4 -hydroxyphenyl)-2-azetidinone (SCH 58235): a designed, potent, orally active inhibitor of cholesterol absorption. 7 Med Chem 1998; 41: 973-80.

12 Van Heek M, France CF, Compton DS et al. In vivo metabolism-based discovery of a potent cholesterol absorption inhibitor, SCH58235, in the rat and rhesus monkey through the identification of the active metabolites of SCH48461.J Pharmacol Exp Ther 1997; 283: 157-63.

13 Altmann SW, Davis HR Jr., Zhu LJ et al. Niemann-Pick G1 Like 1 protein is critical for intestinal cholesterol absorption. Science 2004; 303: 1201-4.

14 Knopfel M, Davies JP, Duong PT et al. Multiple plasma membrane receptors but not NPC1L1 mediate high-affinity, ezetimibe-sensitive cholesterol uptake into the intestinal brush border membrane. Biochim Biophys Acta 2007; 1771: 1140-7.

15 Murdoch D, Scott LJ. Ezetimibe/Simvastatin: a review of its use in the management of hypercholesterolemia. Am $\mathcal{J}$ Cardiovasc Drugs 2004; 4: 405-22.
16 Kosoglou T, Statkevich P, Johnson-Levonas AO et al. Ezetimibe: a review of its metabolism, pharmacokinetics and drug interactions. Clin Pharmacokinet 2005; 44: 467-94.

17 Gustavson LE, Schweitzer SM, Burt DA et al. Evaluation of the potential for pharmacokinetic interaction between fenofibrate and ezetimibe: A phase I, open-label, multipledose, three-period crossover study in healthy subjects. Clin Ther 2006; 28: 373-87.

18 MSD-SP Ltd. Ezetrol $10 \mathrm{mg}$ tablets. Summary of product characteristics. March 2008.

19 van Heek M, Farley C, Compton DS et al. Comparison of the activity and disposition of the novel cholesterol absorption inhibitor, SCH58235, and its glucuronide, SCH60663. Br J Pharmacol 2000; 129: 1748-54.

20 Flores NA. Ezetimibe + simvastatin (Merck/ScheringPlough). Curr Opin Investig Drugs 2004; 5: 984-92.

21 Knopp RH, Gitter H, Truitt T et al. Effects of ezetimibe, a new cholesterol absorption inhibitor, on plasma lipids in patients with primary hypercholesterolemia. Eur Heart $\mathcal{F}$ 2003; 24: 729-41.

22 Dujovne CA, Ettinger MP, McNeer JF et al. Efficacy and safety of a potent new selective cholesterol absorption inhibitor, ezetimibe, in patients with primary hypercholesterolemia. Am 7 Cardiol 2002; 90: 1092-7.

23 Gazi IF, Daskalopoulou SS, Nair DR, Mikhailidis DP. Effect of ezetimibe in patients who cannot tolerate statins or cannot get to the low density lipoprotein cholesterol target despite taking a statin. Curr Med Res Opin 2007; 23: 2183-92.

24 Brohet C, Banai S, Alings AM et al. LDL-C goal attainment with the addition of ezetimibe to ongoing simvastatin treatment in coronary heart disease patients with hypercholesterolemia. Curr Med Res Opin 2005; 21: $571-8$.

25 Farnier M, Volpe M, Massaad R, Davies MJ, Allen C. Effect of co-administering ezetimibe with on-going simvastatin treatment on LDL-C goal attainment in hypercholesterolemic patients with coronary heart disease. Int 7 Cardiol 2005; 102: 327-32.

26 Cruz-Fernandez JM, Bedarida GV, Adgey J et al. Efficacy and safety of ezetimibe co-administered with ongoing atorvastatin therapy in achieving low-density lipoprotein goal in patients with hypercholesterolemia and coronary heart disease. Int $f$ Clin Pract 2005; 59: 619-27.

27 Pearson TA, Denke MA, McBride PE et al. A communitybased, randomized trial of ezetimibe added to statin therapy to attain NCEP ATP III goals for LDL cholesterol in hypercholesterolemic patients: the ezetimibe add-on to statin for effectiveness (EASE) trial. Mayo Clin Proc 2005; 80: 587-95.

28 Garcia-Calvo M, Lisnock J, Bull HG et al. The target of ezetimibe is Niemann-Pick C1-Like 1 (NPG1L1). Proc Natl Acad Sci U S A 2005; 102: 8132-7.

29 Bissonnette S, Habib R, Sampalis F, Boukas S, Sampalis JS. Efficacy and tolerability of ezetimibe $10 \mathrm{mg} /$ day coadministered with statins in patients with primary hypercholesterolemia who do not achieve target LDL-C while on statin monotherapy: A Canadian, multicentre, prospective study - the Ezetrol Add-On Study. Can 7 Cardiol 2006; 22: 1035-44. 
30 Simons LA, Symons J. Ezetimibe added to statin therapy (EASY study) - an evaluation by Australian general practitioners. Aust Fam Physician 2007; 36: 90-2.

31 Ballantyne CM, Houri J, Notarbartolo A et al. Effect of ezetimibe coadministered with atorvastatin in 628 patients with primary hypercholesterolemia: a prospective, randomized, double-blind trial. Circulation 2003; 107: 240915.

32 Davidson MH, McGarry T, Bettis R et al. Ezetimibe coadministered with simvastatin in patients with primary hypercholesterolemia. 7 Am Coll Cardiol 2002; 40: 2125-34.

33 Melani L, Mills R, Hassman D et al. Efficacy and safety of ezetimibe coadministered with pravastatin in patients with primary hypercholesterolemia: a prospective, randomized, double-blind trial. Eur Heart 7 2003; 24: 717-28.

34 Kerzner B, Corbelli J, Sharp S et al. Efficacy and safety of ezetimibe coadministered with lovastatin in primary hypercholesterolemia. Am $\mathcal{J}$ Cardiol 2003; 91: 418-24.

35 Davidson MH, Ballantyne CM, Kerzner B et al. Efficacy and safety of ezetimibe coadministered with statins: randomised, placebo-controlled, blinded experience in 2382 patients with primary hypercholesterolemia. Int 7 Clin Pract 2004; 58: 746-55.

36 Ballantyne CM, Weiss R, Moccetti T et al. Efficacy and safety of rosuvastatin $40 \mathrm{mg}$ alone or in combination with ezetimibe in patients at high risk of cardiovascular disease (results from the EXPLORER study). Am $\mathcal{F}$ Cardiol 2007; 99: 673-80.

37 Ballantyne CM, Lipka LJ, Sager PT et al. Long-term safety and tolerability profile of ezetimibe and atorvastatin coadministration therapy in patients with primary hypercholesterolaemia. Int 7 Clin Pract 2004; 58: 653-8.

38 Blagden MD, Chipperfield R. Efficacy and safety of ezetimibe co-administered with atorvastatin in untreated patients with primary hypercholesterolaemia and coronary heart disease. Curr Med Res Opin 2007; 23: 767-75.

39 Stein E, Stender S, Mata P et al. Achieving lipoprotein goals in patients at high risk with severe hypercholesterolemia: efficacy and safety of ezetimibe co-administered with atorvastatin. Am Heart 7 2004; 148: 447-55.

40 Betteridge DJ. Treating dyslipidaemia in the patient with type 2 diabetes. Eur Heart 7 Suppl 2004; 6: C28-33.

41 Farnier M, Freeman MW, Macdonell G et al. Efficacy and safety of the coadministration of ezetimibe with fenofibrate in patients with mixed hyperlipidaemia. Eur Heart 7 2005; 26: 897-905.

42 McKenney JM, Farnier M, Lo KW et al. Safety and efficacy of long-term co-administration of fenofibrate and ezetimibe in patients with mixed hyperlipidemia. $7 \mathrm{Am}$ Coll Cardiol 2006; 47: 1584-7.

43 Farnier M, Roth E, Gil-Extremera B et al. Efficacy and safety of the coadministration of ezetimibe/simvastatin with fenofibrate in patients with mixed hyperlipidaemia. $\mathrm{Am}$ Heart 7. 2007; 153: 335.el-335.e8.

44 Knopp RH, Tsunehara C, Retzlaff BM et al. Lipoprotein effects of combined ezetimibe and colesevelam hydrochloride versus ezetimibe alone in hypercholesterolemic subjects: a pilot study. Metabolism, Clinical E Experimental 2006; 55: 1697-703.
45 Zema MJ. Colesevelam $\mathrm{HCl}$ and ezetimibe combination therapy provides effective lipid-lowering in difficult-to-treat patients with hypercholesterolemia. Am $\mathcal{F}$ Ther 2005; 12: 306-10.

46 Xydakis AM, Guyton JR, Chiou P et al. Effectiveness and tolerability of ezetimibe add-on therapy to a bile acid resinbased regimen for hypercholesterolemia. Am 7 Cardiol 2004; 94: 795-7.

47 Bays H, Rhyne J, Abby S, Lai YL, Jones M. Lipid-lowering effects of colesevelam $\mathrm{HCl}$ in combination with ezetimibe. Curr Med Res Opin 2006; 22: 2191-200.

48 Lichtenstein AH, Appel LJ, Brands M, et al. Summary of American Heart Association diet and lifestyle recommendations revision 2006. Arterioscler Thromb Vasc Biol 2006; 26: 2186-2191.

49 Sudhop T, Lütjohann D, Kodal A et al. Inhibition of intestinal cholesterol absorption by ezetimibe in humans. Circulation 2002; 106: 1943-48.

50 Assmann G, Kannenberg F, Ramey DR et al. Effects of ezetimibe, simvastatin, atorvastatin, and ezetimibe therapies on non-cholesterol sterols in patients with primary hypercholesterolaemia. Curr Med Res Opin 2007 Nov 30. Source http://libra.publisher.ingentaconnect.com/content/ libra/cmro/pre-prints. Accessed 18 December 2007.

51 Jakulj L, Trip MD, Sudhop T, et al. Inhibition of cholesterol absorption by the combination of dietary planet sterols and ezetimibe: effects on plasma lipid levels. 7 Lipid Res 2005; 46: 2692-2698.

52 Geiss HC, Otto C, Hund-Wissner E, Parhofer KG. Effects of ezetimibe on plasma lipoproteins in severely hypercholesterolemic patients treated with regular LDLapheresis and statins. Atherosclerosis 2005; 180: 107-12.

53 Yamamoto A, Harada-Shiba M, Endo M et al. The effect of ezetimibe on serum lipids and lipoproteins in patients with homozygous familial hypercholesterolemia undergoing LDL-apheresis therapy. Atherosclerosis 2006; 186: 126-31.

54 Gagne C, Gaudet D, Bruckert E. Efficacy and safety of ezetimibe coadministered with atorvastatin or simvastatin in patients with homozygous familial hypercholesterolemia. Circulation 2002; 105: 2469-75.

55 Wierzbicki AS, Doherty E, Lumb PJ, Chik G, Crook MA. Efficacy of ezetimibe in patients with statin-resistant and statin-intolerant familial hyperlipidaemias. Curr Med Res Opin 2005; 21: 333-8.

56 Simons L, Tonkon M, Masana L et al. Effects of ezetimibe added to on-going statin therapy on the lipid profile of hypercholesterolemic patients with diabetes mellitus or metabolic syndrome. Curr Med Res Opin 2004; 20: 1437-45.

57 Pearson T, Denke M, McBride P et al. Effectiveness of the addition of ezetimibe to ongoing statin therapy in modifying lipid profiles and attaining low-density lipoprotein cholesterol goals in older and elderly patients: subanalyses of data from a randomized, double-blind, placebo-controlled trial. Am 7 Geriatr Pharmacother 2005; 3: 218-28.

58 Lipka L, Sager P, Strony J et al. Efficacy and safety of coadministration of ezetimibe and statins in elderly patients with primary hypercholesterolaemia. Drugs Aging 2004; 21 : $1025-32$. 
59 Coll B, Aragones G, Parra S, Alonso-Villaverde C, Masana L. Ezetimibe effectively decreases LDL-cholesterol in HIVinfected patients. Aids 2006; 20: 1675-7.

60 Negredo E, Molto J, Puig J et al. Ezetimibe, a promising lipid-lowering agent for the treatment of dyslipidaemia in $\mathrm{HIV}$-infected patients with poor response to statins. Aids 2006; 20: 2159-64.

61 Bennett MT, Johns KW, Bondy GP. Ezetimibe is effective when added to maximally tolerated lipid lowering therapy in patients with HIV. Lipids Health Dis 2007; 6: 15.

62 Buchanan C, Smith L, Corbett J, Nelson E, Shihab F. A retrospective analysis of ezetimibe treatment in renal transplant recipients. Am $\mathcal{F}$ Transplant 2006; 6: 770-4.

63 Kohnle M, Pietruck F, Kribben A et al. Ezetimibe for the treatment of uncontrolled hypercholesterolemia in patients with high-dose statin therapy after renal transplantation. Am 7 Transplant 2006; 6: 205-8.

64 Patel AR, Ambrose MS, Duffy GA, Cote H, DeNofrio D. Treatment of hypercholesterolemia with ezetimibe in cardiac transplant recipients. 7 Heart Lung Transplant 2007; 26: $281-4$.

65 Bays HE, Moore PB, Drehobl MA et al. Effectiveness and tolerability of ezetimibe in patients with primary hypercholesterolemia: pooled analysis of two phase II studies. Clin Ther 2001; 23: 1209-30.
66 Gagne C, Bays HE, Weiss SR et al. Efficacy and safety of ezetimibe added to ongoing statin therapy for treatment of patients with primary hypercholesterolemia. Am $\mathcal{f}$ Cardiol 2002; 90: 1084-91.

67 Cook JR, Yin D, Alemao E et al. Cost-effectiveness of ezetimibe coadministration in statin-treated patients not at cholesterol goal: application to Germany, Spain and Norway. Pharmacoeconomics 2004; 22 Suppl 3: 49-61.

68 Kohli M, Attard C, Lam A et al. Cost effectiveness of adding ezetimibe to atorvastatin therapy in patients not at cholesterol treatment goal in Canada. Pharmacoeconomics 2006; 24: 815-30.

69 NICE Technology Appraisal Guidance 132. Ezetimibe for the treatment of primary (heterozygous - familial and non familial) hypercholesterolaemia. www.nice.org.uk

Correspondence:

Stephen I'Anson, Just Medical Media Ltd, 11 Redgrove Park, Cheltenham, Gloucestershire, GL51 6QY, UK.

Email: publisher@justmedicalmedia.com. 
